\title{
La formación del profesorado en educación física con relación a las personas con discapacidad
}

\section{The teacher training on physical education for persons with disability}

\section{RESUMEN}

En el ámbito educativo, uno de los temas más controvertidos es la inclusión de alumnos con discapacidad en los centros educativos ordinarios, tanto en primaria como en secundaria.

Los mayores obstáculos y dificultades que señalan los docentes para la aplicación de la inclusión educativa alude a la falta de recursos materiales y personales, a la heterogeneidad de los alumnos en los grupos de clase y a la falta de preparación para la atención a la diversidad, provocando todo ello un amplio malestar entre el profesorado.

La inclusión debemos plantearla desde un modelo ecológico (Hutzler, 2005) donde no sólo se basa en la instrucción si no en la tarea, en el entorno, en el patrón de movimiento y en el alumno para poder llevar a cabo la habilidad prevista.

\section{PALABRAS CLAVE}

Atención a la diversidad, Formación, Educación fisica, Inclusión.

\section{ABSTRACT}

In the educational area, one of the most controversial topics is the inclusion of students with disability in the educational ordinary school, so much in primary as in secondary education.

The main obstacles and difficulties indicated by the teachers are the lack of material

* Directora Gerente del Centro de referencia Estatal para la Atención a Personas con Grave Discapacidad y para la Promoción de la autonomía Personal y Atención a la Dependencia. 
and personal resources, related to the students heterogeneity in the different class group as well as the absence of preparation in the attention to the diversity. This inclusion must be raised from an ecological model (Hutzler, 2005) not only based on instruction, but in task, in environment, in movement and in the student to carry out the skill.

\section{KEY WORDS}

Attention to the diversity, Training, Physical education teacher, Inclusion. 
En el ámbito educativo, uno de los temas más controvertidos es la inclusión de alumnos con discapacidad en los centros educativos ordinarios, tanto en primaria como en secundaria.

Se han ido publicando diferentes textos normativos (LOGSE, LOCE y finalmente la LOE), pero en numerosas ocasiones la inclusión ha quedado sobre el papel, sin que realmente el profesorado disponga de recursos y herramientas para hacer viable una inclusión que precisa de una serie de adaptaciones para esas necesidades educativas especificas (nee) que presentan algunos alumnos.

Se debe concebir la inclusión como un principio y no como una medida que corresponde a unos pocos. "No sólo se trata de promover procesos de enseñanza $y$ aprendizaje para un conjunto específico de estudiantes que aprenden unos determinados contenidos, conceptos, procedimientos y lleguen a asumir de manera reflexiva un sistema de valores; sino asimismo, de motivar y desarrollar todo un conjunto de destrezas que les permitan establecer nuevas relaciones $e$ intercambios con esos y otros contenidos culturales" (Torres, 1999).

El dilema que se plantea actualmente en el Sistema Educativo en España no es otro que saber si la exigencia de igualdad en el grupo-clase no estará deteriorando la calidad educativa en el aula. La dotación de mayores recursos educativos (número de profesores o especialistas, ampliación del material didáctico, formación del profesorado, capacidad para elaborar proyectos educativos y curriculares flexibles, nuevas formas de organización escolar, eficaz coordinación entre distintos profesionales, eficacia en la orientación educativa,...) es requisito necesario para atender a la diversidad.
Los mayores obstáculos y dificultades que señalan los docentes para la aplicación de la inclusión educativa alude a la falta de recursos materiales y personales, a la heterogeneidad de los alumnos en los grupos de clase y a la falta de preparación para la atención a la diversidad, provocando todo ello un amplio malestar entre el profesorado.

No obstante, la complejidad y la magnificencia de dichos problemas no ha de suponer una merma en el buen hacer de los futuros profesionales, que con la formación adecuada deben luchar para conseguir solventar los obstáculos actuales, en un intento más por hacer realidad el sueño de la inclusión educativa.

\section{LA SITUACIÓN ACTUAL DE LA INCLUSIÓN EDUCATIVA EN EDUCACIÓN FÍSICA}

La situación actual de la educación fisica frente a la atención de acnee, presenta un panorama no excesivamente alentador como nos muestran los datos de algunas investigaciones que se detallarán a continuación.

Desde hace tiempo, se necesita una respuesta urgente a la situación actual de la atención a la diversidad del alumnado que se encuentran en las aulas y que no es posible mantener dentro del enfoque tradicional. Se ha observado una mejora en la inclusión en la etapa de Primaria ya que los profesores están más preparados y más concienciados en relación a la atención a la diversidad y los propios alumnos observan ya como "normal" el compartir sus aulas con alumnos con discapacidad.

La escuela ordinaria concibe la educación como destinada al alumno medio, por lo que muchos alumnos quedan sin 
la debida atención por la rigidez de la organización, de los espacios y de los tiempos. En esta concepción, las diferencias individuales son consideradas como retraso en el desarrollo, lagunas en el aprendizaje, alteraciones en el desarrollo de la personalidad o problemas derivados de la situación familiar. Atendiéndose más al déficit que a la necesidad y más a lo que el alumno tiene de distinto que a lo que tiene de igual (Ríos, 2003).

Si se acepta que la educación tiene los mismos fines para todos, todos son todos $\mathrm{y}$, desde el sistema educativo, tiene que posibilitarse a cada individuo que alcance el grado de consecución de los fines de acuerdo con sus capacidades, con las posibilidades del medio y con los recursos que él necesite. Hay que tener presente que la inclusión no sólo beneficia a los acnees sino a todos los que están a su alrededor: compañeros de clase, profesores, etc., y también, al centro educativo.

Estudios realizados, tanto en Primaria como en Secundaria, demuestran como un elevado porcentaje de profesores de Educación Física no atienden a la diversidad tal y como refleja la Ley (Hernández, 2000). Así se expone que, en general, el profesorado no tiene la preparación suficiente para atender a los alumnos con nee y que los cursos de formación deberían cubrir aspectos tales como la metodología e intervención didáctica (Sanz, Reina, Mendoza, 2001). El profesorado se escuda en la falta de conocimientos para no cometer errores en su intervención y así no intervenir. Ese miedo a actuar por desconocimiento da lugar a la inactividad o deficitaria actividad física desarrollada por el alumno con nee en las clases de Educación Física. De hecho, algunos factores claves que condicionan que el objetivo de la inclusión sea difícil de alcanzar son (Calverol, 2000):
1. Las barreras arquitectónicas en los centros de enseñanza.

2. La formación e información del profesorado de Educación Física, así como el aislamiento ante un problema sin contar con un profesional de apoyo.

Pelechano (1989) y Aguado y Alcedo (1991), coinciden en que las actitudes ante las personas con discapacidad son actualmente negativas. Algunos autores como Aguado y Alcedo (1991), estudiaron el tratamiento de los medios de comunicación hacia la discapacidad. El estudio muestra la existencia de actitudes negativas por parte de la sociedad y la utilización de una terminología inadecuada y de prejuicios y estereotipos establecidos. Verdugo y Arias (1991), trabajan en investigaciones realizadas en varios países sobre el tema de las actitudes hacia las personas con discapacidad.

Verdugo, Jenaro y Arias (1994), hacen un estudio donde construyen un instrumento de medida en forma de escala sobre las actitudes hacia las personas con discapacidad (Escala General de Actitudes). Escandell y Santiago (1998), realizan un estudio donde señalan algunos aspectos que inciden en las actitudes en la integración de sujetos con necesidades educativas especiales.

En el ámbito de la educación, uno de los primeros trabajos sobre las actitudes del profesorado hacia la integración escolar fue el elaborado por García y Alonso (1985), donde se aborda el estudio de diferentes variables relacionadas con las actitudes, algunas sobre la discapacidad.

Abós y Polaino (1986), publican un trabajo en el que hacen referencia a varios factores que tienen relación con la integración escolar, como la filosofia de la integración, los efectos sobre el niño 
deficientes y no deficientes, la formación y conducta docente y las condiciones de la integración. Sáenz (1990), hace una revisión de las principales investigaciones de los últimos años referidas a las actitudes del profesorado hacia la integración escolar, dedicando una atención principal a buscar acciones para reducir la resistencia a la integración.

García, García y Rodríguez (1991), destacan la importancia del profesor para que haya un mayor desarrollo de las actitudes positivas en torno a la integración escolar. En este sentido, también Dengra, Durán y Verdugo (1991), realizan un estudio sobre la influencia de las variables que inciden en la formación del profesorado, para que actúe de forma positiva en la integración escolar.

Gozalo (1996), muestra un estudio sobre las actitudes, tratando de ver las diferencias y semejanzas de las actitudes que aparecen entre los profesionales que se dedican específicamente a la atención de alumnos con discapacidad. Sales, Moliner y Sanchiz (2001), plantean la necesidad de desarrollar actitudes positivas hacia la diversidad, partiendo de una formación inicial del profesorado. Elaboran un cuestionario para conocer las actitudes, y se advierten actitudes negativas hacia la inclusión y la diversidad.

En el ámbito de la Educación Física hay estudios como el de Cid (2003), que realiza una investigación sobre las actitudes de niños y niñas de primaria hacia sus compañeros con discapacidad en las clases de educación física. En general las actitudes hacia el alumnado con discapacidad son positivas, pero pueden estar condicionadas por sus orientaciones hacia determinados modelos de participación social en el aprendizaje. Reina (2003), hace una reflexión en torno a las actitudes manifestadas hacia las personas con discapacidad a través de las actividades deportivas y recreativas, y propone una serie de pautas para la modificación de las actitudes hacia ese colectivo, en experiencias de contacto directo con personas con discapacidad.

Los valores son un componente de las actitudes, siendo las actitudes componentes más generales y los valores serían las apreciaciones que un individuo hace de un objeto. Existe una gran relación entre actitudes y valores. "Toda actitud está relacionada con unos valores determinados, o todo valor implica una o más actitudes concretas" (Prat y Soler, 2003).

Antes de continuar, apuntar, siguiendo a Arráez (2003) que cuando se habla de colectivos especiales, se esta haciendo referencia no solamente a las personas con discapacidad sino también a personas con alteraciones fisiológicas (cardiopatías, asma, diabetes, etc.), personas mayores y, sobretodo, a los colectivos emergentes que no son otros que, colectivos que por causas personales o ambientales negativas, no pueden desarrollar su potencial personal de manera normal y que, además, no reciben atención preferente por parte de las instituciones, al igual que se presta a otros colectivos en desventaja (Arráez, 2003).

En esta misma línea, Hernández (1998) realiza un estudio sobre la situación del profesorado de EF en primaria y llega a la conclusión que, existe una actitud positiva hacia la integración pero reconoce las limitaciones pedagógicas para poder atender a la diversidad lo que supone un déficit formativo en los docentes.

Ríos (1999, 2001, 2003) nos habla de diferentes factores que influyen en el proceso de inclusión de los acnee, como son: condicionantes infraestructurales, sociales, de los propios alumnos y de la práctica docente. 
Ruiz Sánchez (2000) nos propone propuestas para mejorar la inclusión, trabajando en una mayor formación universitaria específica de esta área, profesores de apoyo, mejora de los recursos humanos, elaborar un cuaderno de registro del alumno para toda su escolarización, etc.

Arráez (1998) nos ofrece una serie de consideraciones a tener en cuenta, en el tratamiento de estos alumnos con nee:

- Se debe crear cuanto antes un clima de aceptación normal, de agradable y amistosa convivencia.

- Tratar de lograr la aceptación de sí mismo como condición previa e indispensable para adquirir un equilibrio emocional, afectivo y social adecuado.

- Insistir en que cada alumno, dentro de sus limitaciones, consiga el mayor grado de independencia y autonomía posible, para la transferencia que esto le pueda suponer a su vida diaria.

Una vez revisado la evolución del proceso normativo por lo que respecta a la integración de alumnos con nee en el centro escolar, podemos definir la integración, tal y como indica Birch (1974, en Bautista, 1993) como "un proceso que pretende unificar las educaciones ordinaria y especial con el objetivo de ofrecer un conjunto de servicios a todos los niños, en base a sus necesidades de aprendizaje". Sanz y Reina (2003) apuntan que en el sistema educativo español existen diferentes tipos de integración, que estarán en función, tanto de las características del niño con necesidades educativas especiales como de las posibilidades de la Escuela o Centro en el que vaya a ser integrado. En este sentido, nos encontramos con diferentes modalidades de integración: a) Integración Completa, en la que el niño asiste al colegio normal y al aula normal según su edad, pero recibe alguna ayuda de cara a la realización de determinadas actividades más elaboradas para superar pequeñas dificultades o signos de inmadurez, normalmente pasajeros.

b) La Integración Combinada supone que el niño asiste al colegio normal y a la clase normal según su edad, pero recibe durante un tiempo diario más o menos largo, dependiendo de sus necesidades, algunas sesiones con un profesor especialista.

c) La Integración Parcial supone que el niño asiste al colegio normal, pero recibe su escolarización en el aula especial al frente de la cual hay un profesor de Educación Especial. No obstante, estos niños participan en el resto de la vida habitual del resto de los niños y niñas dentro del centro escolar.

d) La Integración de Centro Específico, que representa aquella en la que el niño no asiste al colegio normal, sino que su escolaridad se le imparte en jornada completa en un colegio especial.

Entendemos, por lo tanto, que el sistema educativo, en su conjunto, debe proveer los medios necesarios para proporcionar la ayuda que cada alumno necesite, dentro de un contexto educativo lo más normalizado posible y adaptándose tanto a las características que presente el alumnado como a las posibilidades que disponga el Centro.

\section{ESTRATEGIAS DE INTERVENCIÓN DEL PROFESORADO DE EDUCACIÓN FÍSICA}

Una vez conocida la situación actual 
de nuestro área sobre la inclusión de colectivos especiales y emergentes, se debe llegar a plantearse una forma de intervención para mejorar nuestra situación actual. Al analizar lo que ocurre en los centros de enseñanza, se observa que cada profesor va utilizando las herramientas y recursos de los que dispone para poder hacer frente a la inclusión de acnee al no existir recetas debido a la variedad y la heterogeneidad de las diferentes discapacidades y colectivos emergentes. Se ha percibido que cuanta mayor es la formación del profesorado mayor cantidad de respuestas educativas puede ofrecer al alumno con nee. También, hay que tener presente que no puede ir el área de EF aislada para lograr la inclusión del alumnado sino que todo el centro debe ir en bloque buscando el mismo objetivo: la inclusión.

El programa de intervención, que se está llevando a cabo actualmente en casi todos los centros, es el relacionado con la sensibilización de los alumnos, donde todos los alumnos, independientemente de tener 0 no discapacidad 0 pertenecer a colectivos emergentes, vivenciarán una situación determinada que les limite su funcionalidad habitual. Esta limitación artificial ayudará a educar a nuestros alumnos en valores y actitudes hacia las personas con discapacidad y personas de colectivos especiales, puesto que el hecho de vivenciar e identificar una situación limitante a la que no están acostumbrados les ayudará a valorar y entender las posibles dificultades de los compañeros que tengan una discapacidad. Ríos, (2003) afirma que "el estudio de casos también gana protagonismo, simulando situaciones de grupo específico (formado solo por personas con la misma discapacidad) o de inclusión (con la presencia de personas con y sin discapacidad), es decir, hacer un trabajo reflexivo sobre la acción, de tal manera que el propio alumnado consigue la asimilación de contenidos teóricos, adaptando las tareas según las características del alumno o alumna que simula una discapacidad, sin obviar las necesidades educativas de todo el grupo. Es mucho más significativo una sesión donde los alumnos se sienten en una silla de ruedas y visiten su centro para comprobar las barreras arquitectónicas que presenta el lugar que una sesión de diapositivas con relación a las barreras arquitectónicas".

No se obvia que el hecho de atender a las nee supone un esfuerzo extra para el profesor, puesto que, en ocasiones, no es fácil solucionar la situación atendiendo a la diversidad partiendo del criterio fundamental que para atender a los acnee se adapte sólo lo que sea necesario sin desvirtuar la esencia de la actividad y así mantener la calidad docente que se pretende (Ríos, 2003).

Para que la inclusión escolar se desarrolle con éxito se deben procurar unas condiciones precisas (Bautista, 1993):

- El establecimiento de programas y experiencias piloto que preceden a cualquier generalización de los procedimientos inclusivos.

- La realización de campañas de información y mentalización que preparen adecuadamente el terreno para la inclusión.

- Una legislación que garantice y facilite la misma.

- Cambios y renovación de la escuela tradicional referidas a su organización, estructura, metodologia, objetivo, etc.

- Reducción de la proporción profesor/alumnos por aula. 
- Un diseño curricular único, abierto y flexible.

- Supresión de barreras arquitectónicas y adaptación de centros ordinarios.

- Recursos personales, materiales y didácticos adecuados.

- Participación activa de los padres.

- La formación y perfeccionamiento del profesorado.

La formación y perfeccionamiento del profesorado. Sanz (03) nos muestra como Jansma, P., y Surburg, P. (1995) realizaron durante seis años un estudio para definir las competencias que debiera reunir un profesional de la educación física adaptada, en un programa específico de formación. Realizaron un listado de 79 competencias, donde se ponen como ejemplo:

- Adquirir un conocimiento básico respecto a los contenidos desarrollados en los cursos de actividad física adaptada.

- Demostrar un amplio conocimiento sobre las condicionantes de la discapacidad, el rendimiento motor y fisico de las personas con discapacidad, valoración de recursos y modelos curriculares en educación física adaptada.

- Demostrar conocimientos avanzados en torno a la legislación referente a la educación con personas con discapacidad.

- Adquirir conocimientos para una intervención temprana en preescolares con discapacidad, conociendo el rol que debe desarrollar el educador para esta población.
- Demostrar habilidad para formular planes curriculares adaptados.

- Desarrollar habilidades para realizar evaluaciones y cursos para futuros profesionales en el ámbito de la actividad física adaptada.

- Identificar patrones normales o retrasos, en el desarrollo motor, la postura, el juego, la condición física, para establecer relaciones con la afectación que pueda tener.

- Demostrar un conocimiento amplio de la literatura existente respecto de la actividad física adaptada.

- Evaluar la calidad y cantidad de los aprendizajes realizados por los alumnos durante el proceso de intervención didáctica.

- Utilizar múltiples recursos profesionales y medios para mejorar las técnicas de intervención, metodologias, y programas.

Sanz (06) destaca que los objetivos que se deberian tener presentes en los cursos básicos de formación de profesores de educación física ó de técnicos deportivos serian:

- Proporcionar a los alumnos los conocimientos básicos para atender a las necesidades educativas especiales que pueda presentar los alumnos con discapacidad.

- Mostrar las diferentes prácticas deportivas que pueden realizar las personas con discapacidad.

- Dar a conocer las principales adaptaciones que se pueden realizar durante el desarrollo de las actividades: materiales, técnicas, tácticas, reglamentarias, etc. 
- Identificar, mediante la vivenciación de situaciones limitantes, las dificultades para realizar ciertas actividades.

- Dar a conocer los principios básicos de las clasificaciones deportivas para personas con discapacidad.

- Identificar el marco legal y federativo que regula el deporte adaptado.

La inclusión debemos plantearla desde un modelo ecológico (Hutzler, 2005) donde no sólo se basa en la instrucción si no en la tarea, en el entorno, en el patrón de movimiento y en el alumno para poder llevar a cabo la habilidad prevista. Se debe:

- Identificar los objetivos de las tareas.

- Establecer los criterios de rendimiento en los que basarse (cualitativos y cuantitativos).
- Detectar los factores que limiten y permitan la actuación, intentando priorizar lo que sí puede hacer ante lo que no puede hacer.

- Observar los resultados y ante los errores que aparecen intentar solucionarlos para futuras intervenciones.

- Manipular los criterios de adaptación que serán los parámetros de control (tarea, entorno, equipamiento, metodologia, reglas, etc.)

Un ejemplo de esta teoría se puede observar en el siguiente caso: una persona con parálisis cerebral que debe realizar una serie de movimientos rítmicos durante un tiempo determinado. Se deben tener en cuenta los siguientes aspectos para poder llevar a cabo la adaptación:

\begin{tabular}{|l|l|l|l|}
\hline CRITERIOS & LIMITACIONES & SITUACION ACTUAL & MODIFICACIONES \\
\hline Posición básica de pie & $\begin{array}{l}\text { Incapaz de } \\
\text { mantenerse de pie sin } \\
\text { ayuda de sus muletas }\end{array}$ & No puede estar de pie & $\begin{array}{l}\text { Tumbada o en } \\
\text { posición de rodillas }\end{array}$ \\
\hline Ejercicios de piernas & $\begin{array}{l}\text { Movimientos } \\
\text { limitados en piernas, } \\
\text { cadera y rodillas. }\end{array}$ & $\begin{array}{l}\text { Rango de movimiento } \\
\text { reducido y diferente } \\
\text { fuerza en los } \\
\text { movimientos de }\end{array}$ & $\begin{array}{l}\text { Uso de movimientos } \\
\text { bilaterales y } \\
\text { activación del tronco } \\
\text { (rodar por el suelo) }\end{array}$ \\
\hline $\begin{array}{l}\text { Ejercicios de brazos } \\
\text { pinguno en tobillos } \\
\text { brazos y piernas }\end{array}$ & $\begin{array}{l}\text { Reducida un } 80 \% \text { de } \\
\text { movilidad en } \\
\text { hombros y nula } \\
\text { coordinación }\end{array}$ & $\begin{array}{l}\text { Función del brazo } \\
\text { limitado, a veces } \\
\text { necesita mantener el }\end{array}$ & $\begin{array}{l}\text { Realizar los } \\
\text { movimientos de } \\
\text { brazos en otra }\end{array}$ \\
\hline contraliatiorio de pie & $\begin{array}{l}\text { posición que no sea } \\
\text { de pie }\end{array}$ \\
\hline
\end{tabular}


Según Pérez, López e Iglesias (2004) se deben mantener los programas con contenidos similares y adaptar las actividades que desarrollan esos contenidos. Hacer más hincapié en aquellos contenidos donde este alumnado pueda incluirse y participar sin demasiadas adaptaciones. Se intentará trabajar más actividades que promuevan una colaboración mutua, juegos cooperativos y menos actividades competitivas. Con estas medidas se busca un mayor tiempo de compromiso motor, un clima positivo de aula, una adecuada organización y control, una óptima información previa y un buen feedback. Variando el tipo de ejecución del grupo, ya sea simultánea (todos al mismo tiempo), alternativa (por pare- jas) o consecutiva (subgrupos de tres) se conseguirá facilitar la inclusión por lo tanto, se debe combinar las diferentes formas de agrupamientos de alumnos.

Finalizar con una de las conclusiones de Pérez, López e Iglesias (2004) en su trabajo sobre la atención a la diversidad en el área de educación física en donde manifiestan después de luchar por lograr la inclusión en su aula que: "Si tuviera que resumir este año, diría que es el año en el que probablemente más frustrada y fracasada me haya sentido como maestra, pero también es verdad que es el año en el que más he aprendido y que ahora, echando la vista atrás, me siento más realizada y enriquecida".

\section{BIBLIOGRAFÍA}

Abos, P. y Polaino, A. (1986). Integración de deficientes educables: un estudio de actitudes docentes. Revista Española de Pedagogía, 172, 194-206.

Aguado, A.L. y Alcedo, M.A. (1991). Tratamiento de la discapacidad en la prensa asturiana. Psicothema, 3 (1), 175-198.

Arnáiz Sánchez, P. y Ortiz González, Mํ.C. (1997). El derecho a una educación inclusiva. En A. Sánchez Palomino y J.A. Torres González (Coord.). Educación Especial I. Una perspectiva curricular, organizativa y profesional (pp. 191-207). Madrid: Pirámide.

Bautista, R. (1993). Una escuela para todos: la integración escolar. En R. Bautista Necesidades educativas especiales (pp. 23-37). Málaga: Aljibe.

Arráez, JM (1998). Teoría y praxis de las adaptaciones curriculares en la educación fisica. Un pro- grama de intervención motriz aplicado en la educación primaria. Ed. Aljibe, Málaga.

Arraéz, JM (2003). Intercultura, educación fisica y deporte. Libro de actas del Congreso de Educación física y deporte. Valladolid.

Calverol, T. (2000). Atención a las nee de los alumnos con discapacidad fisica en los Institutos de Educación Secundaria de la ciudad de Barcelona. Apunts, 37-44, 2ํㅡㄹ trimestre $\mathrm{n}^{\circ}$ 60, Barcelona.

Cid, L. (2003). Actitudes de los niños y niñas de primaria hacia sus compañeros con discapacidad en las clases de educación fisica. Revista Tándem. Didáctica de la Educación Física, 11, 79-90.

Dengra, R., Durán, R. y Verdugo, M.A. (1991). Estudio de las variables que afectan a las actitudes de los maestros hacia la integración escolar de niños con necesidades especiales. En Anuario Español e Iberoamericano de Investigación en Educación Especial (pp. 47-48). Madrid: CEPE. 
García, J.N. y Alonso, J.C. (1985). Actitudes de los maestros hacia la integración escolar de niños con necesidades especiales. Infancia y aprendizaje, 30, 51-68.

García, M., García, J.N. y Rodríguez, C. (1991). Actitudes de los maestros hacia la integración. Siglo Cero, 138, 46-51.

García Sánchez, J.N., García Cabero, M., García González, Maㅗ J., y Rodríguez Bravo, C. (1992). Modificación de actitudes de los estudiantes de magisterio hacia la integración escolar de alumnos con necesidades educativas especiales. En Anuario Español e Iberoamericano de Investigación en Educación Especial (pp. 13-55). Madrid: CEPE.

Gozalo Delgado, M. (1996). Evaluación de las actitudes hacia la integración escolar en una muestra de maestros en formación. En M. Marín Sánchez (Comp.), Sociedad y Educación (pp. 479-488). Sevilla: Eudema.

Hernández (1998). Educación fisica especial: actitud $y$ formación d e los docentes en primaria. Apunts, 51, 70-78. Barcelona.

Hernández, J. (2000). La iniciación a los deportes desde su estructura y dinámica. Aplicación a la Educación Física Escolar y al entrenamiento deportivo Capitulo VI, (pp.153-174). Barcelona: INDE.

Hernández, F.J. (2000). El deporte para atender la diversidad: deporte adaptado y deporte inclusivo. Apunts, 60, pp. 46-53.

Jansma, P., Surburg, P. (1995). Competency Guidelines and Adapted Physical Education Profes sional Preparation in the United Status. Adapted Physical Activity Quaterly. 12, 307-322.

Mendoza, N. (2004) Integración escolar. Adaptaciones curriculares en EF. Revista Pedagógica ADAL. Comunidad de Madrid.

Pelechano, V. (Dir.) (1989). Aceptación, habilidades sociales y motivación en la integración de niños ciegos - Informe técnico. Tenerife: Departamento de Personalidad, Universidad de La Laguna.

Prat Grau, M. y Soler Prat, S. (2003). Actitudes, valores y normas en la Educación Física y el deporte. Barcelona: Inde.

Pérez, D., López, V., Iglesias, P. (2004). La atención a la diversidad en educación fisica. Sevilla: Wanceulen, Ed. Deportiva, s.l.
Reina, R. (2003). Propuesta de intervención para la mejora de las actitudes hacia las personas con discapacidad a través de actividades deportivas y recreativas. Revista Digital. Buenos Aires. Año 9, 59. Abril. http://www.efdeportes.com

Ruiz Sánchez, P. (2000). La motricidad y las nee: análisis de una realidad y politicas de actuación. Ponencia del I Congreso de AEMNE. Granada.

Ríos, M. (2003). Manual de educación física adaptada al alumnado con discapacidad. Barcelona: Paidotribo

Sáenz Barrio, O. (1990). Actitudes de los profesores ante la integración del niño discapacitado en la escuela ordinaria: una visión desde la literatura científica. Revista Universitaria de Formación del Profesorado, 8, 135-150.

Sales Ciges, A., Moliner García, O. y Sanchiz Ruiz, M.L. (2001). Actitudes hacia la atención a la diversidad en la formación inicial del profesorado. Revista Electrónica Interuniversitaria de Formación del profesorado, 4 (2). En http://www.aufop.org/ publica/reifp/01v4n2.asp

Sanz, D.; Reina, R.; Mendoza, N. (2001). Una propuesta de intervención en Educación Física para alumnos con necesidades educativas especiales bajo un enfoque sensibilizador. La enseñanza de la Educación Física y el deporte escolar. Actas del IV Congreso Internacional. Santander.

Sanz, D., Reina,R. (2003): Apuntes del curso de Actividad Física y Deporte Adaptado para personas con discapacidad. Manuscrito sin publicar.

Sanz, D. (2006) (en prensa) La formación en actividad fisica adaptada a través de los recursos proporcionados por la red. Propuestas prácticas de educación física inclusiva para la etapa de Secundaria. Mendoza, N. Barcelona: INDE. (Documento pendiente de publicación).

Torres González, J.A. (1999). Educación y diversidad. Bases didácticas y organizativas. Málaga: Aljibe.

Verdugo, M.A. y Arias, B. (1991). Evaluación y modificación de las actitudes hacia los minusválidos. Revista de Psicologia General y Aplicada, 44 (I), 95-102.

Verdugo, M. A., Jenaro, C., y Arias, B. (1994). Actitudes hacia las personas con minusvalia. Madrid: Ministerio de Asuntos Sociales: INSERSO. 